\title{
Decrease in Muscle Contraction Time Complements Neural Maturation in the Development of Dynamic Manipulation
}

\author{
Sudarshan Dayanidhi, ${ }^{1}$ Jason J. Kutch, ${ }^{1}$ and Francisco J. Valero-Cuevas ${ }^{1,2}$ \\ ${ }^{1}$ Division of Biokinesiology and Physical Therapy, University of Southern California, Los Angeles, California 90089, and ${ }^{2}$ Department of Biomedical \\ Engineering, University of Southern California, Los Angeles, California 90089
}

Developmental improvements in dynamic manipulation abilities are typically attributed to neural maturation, such as myelination of corticospinal pathways, neuronal pruning, and synaptogenesis. However the contributions from changes in the peripheral motor system are less well understood. Here we investigated whether there are developmental changes in muscle activation-contraction dynamics and whether these changes contribute to improvements in dynamic manipulation in humans. We compared pinch strength, dynamic manipulation ability, and contraction time of the first dorsal interosseous muscle in typically developing preadolescent, adolescent, and young adults. Both strength and dynamic manipulation ability increased with age $(p<0.0001$ and $p<0.00001$, respectively). Surprisingly, adults had a 33\% lower muscle contraction time compared with preadolescents $(p<0.01)$, and contraction time showed a significant $(p<0.005)$ association with dynamic manipulation abilities. Whereas decreases in muscle contraction time during development have been reported in the animal literature, our finding, to our knowledge, is the first report of this phenomenon in humans and the first finding of its association with manipulation. Consequently, the changes in the muscle contractile properties could be an important complement to neural maturation in the development of dynamic manipulation. These findings have important implications for understanding central and peripheral contributors to deficits in manipulation in atypical development, such as in children with cerebral palsy.

\section{Introduction}

Dexterous manipulation capabilities have a prolonged period of development during childhood (Forssberg et al., 1991) and adolescence (Vollmer et al., 2010; Dayanidhi et al., 2013). These improvements have been primarily attributed to neural factors, including developmental plasticity of the corticospinal tract (Armand et al., 1997; Olivier et al., 1997; Martin, 2005) via microstructural changes (Lebel et al., 2008), myelination (Paus et al., 1999), and synaptogenesis (Giedd et al., 1999). However, the contribution of developmental changes in the muscular system, which would serve to increase the fidelity of the muscular response to neural input, are less well understood.

Children exhibit dramatic increases in hand strength after the age of 10 (Häger-Ross and Rösblad, 2002), attributed to changes in physiological cross-sectional area, moment arm length, and

Received May 7, 2013; revised Aug. 13, 2013; accepted Aug. 15, 2013.

Author contributions: S.D., J.J.K., and F.J.V.-C. designed research; S.D., J.J.K., and F.J.V.-C. performed research; S.D. and J.J.K. contributed unpublished reagents/analytic tools; S.D., J.J.K., and F.J.V.-C. analyzed data; S.D., J.J.K., and F.J.V.-C. wrote the paper.

The contents of this research paper were in part developed under the Department of Education, NIDRR Grant H133E080024 and NSF Grant EFRI-COPN 0836042, and National Institutes of Health Grants AR050520 and AR052345 to F.J.V.-C. However, those contents do not necessarily represent the policy of the Department of Education, and you should not assume endorsement by the Federal Government. We thank the subjects and their families for participating; Terry McKiernan, Susan Smith and Joe Cocozza for recruitment; and Harjot Hansra, Wenhsin Hu, Analiese DiConti, and Jonathan Lerner for their assistance with data collection. S.D. is currently a postdoctoral fellow at University of California, San Diego.

F.J.V.-C. holds US Patent No. 6,537,075 on some of the technology used but has no active or pending licensing agreements with any commercial entity. The other authors declare no competing financial interests.

Correspondence should be addressed to Dr. Francisco J. Valero-Cuevas, University of Southern California, 3710 McClintock Avenue, RTH 404, Los Angeles, CA 90089-2905. E-mail: valero@usc.edu.

DOI:10.1523/JNEUROSCI.1968-13.2013

Copyright $\odot 2013$ the authors $\quad 0270-6474 / 13 / 3315050-06 \$ 15.00 / 0$ activation level (O'Brien et al., 2010). Although strength clearly plays a role in manipulation, most manipulation activities require only low or modest strength levels (Smaby et al., 2004). Dynamic manipulation critically requires that hand muscles faithfully respond to commands from the nervous system, enabling the rapid regulation of fingertip force vector magnitudes and directions based on task requirements (Valero-Cuevas et al., 2003; Venkadesan and Valero-Cuevas, 2008). Consequently, the activation-contraction dynamics of intrinsic hand muscles, defining the time needed for the muscle to respond to neural input, may be an important limiting factor in the performance of dynamic manipulation.

Activation-contraction dynamics can be succinctly described in terms of contraction time, which is a property of single motor units and defines the time required to achieve maximum force after a single action potential in the motor unit of interest. Motor units in intrinsic hand muscles of adult humans have contraction times on the order of $50 \mathrm{~ms}$ (Thomas et al., 1990; Chan et al., $2001)$ and upward of $50 \%$ are recruited, even for low force $(\sim 2 \mathrm{~N})$ activities (Milner-Brown et al., 1973b). In animal models, contraction time decreases by $50-80 \%$ with age (Close, 1964), which is likely the result of improved $\mathrm{Ca}^{2+}$ uptake by the sarcotubules (Brody, 1976) mediated by increased parvalbumin concentration (Heizmann et al., 1982; Leberer and Pette, 1986). Direct measurements of motor unit contraction time in the hand muscles of children have not been made, to our knowledge, likely because of the invasive nature of the required intramuscular or intraneural electrodes in the upper limb and hand. Therefore, the relationship between developmental changes of contraction time and dynamic manipulation is not known. 
A
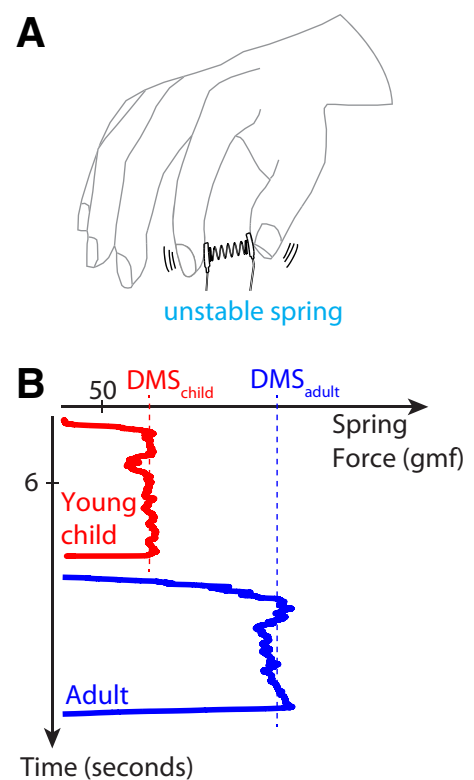

C

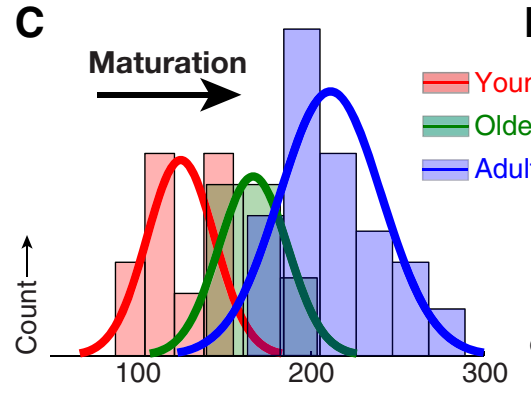

Dynamic manipulation score (DMS - gmf)
D

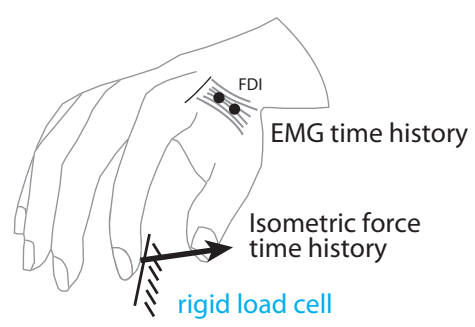

E

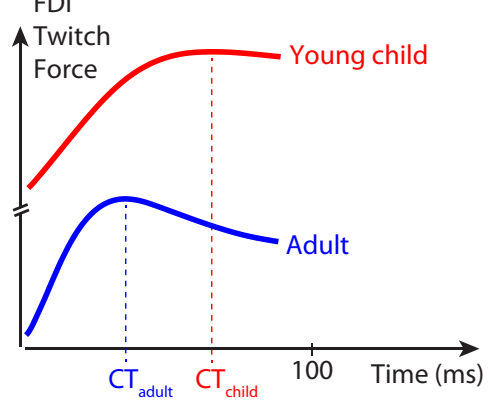

Figure 1. Summary of experiments and results. $A$, Dynamic manipulation ability was tested by compressing and maintaining a low force instrumented spring that was prone to instabilities for at least $3 \mathrm{~s}$. $\boldsymbol{B}$, Representative data show increased maximal sustained compression, i.e. Dynamic Manipulation Score (DMS) in adults (blue) compared with children (red). D, To calculate the muscle contraction time (in terms of average time to peak of force twitch), subjects were asked to maintain $2 \mathrm{~N}$ of isometric force against a rigid 6-axis load cell while surface EMG was collected simultaneously from the first dorsal interosseous. $E$, Representative EWA waveforms are shown for adults (blue) and children (red) with the contraction time indicated. $\boldsymbol{C}, \boldsymbol{F}$, Histograms of DMS and mean contraction times (CT) for all subjects.

Consequently, the goal of this study was to address this impasse by using a recently developed noninvasive technique (Kutch et al., 2010), suitable for use in children, for estimating contraction time in the whole muscle to link fundamental muscle activation-contraction properties with dynamic manipulation performance. We tested for changes in muscle contraction time during the preadolescent and adolescent years and examined whether these changes are associated with improvements in dynamic manipulation capabilities. Because of the prior work in animal models, we hypothesized that contraction time decreases in intrinsic hand muscles during human development, and that this decrease could, in part, explain improvements in dynamic manipulation abilities.

\section{Materials and Methods}

Thirteen adults (ages 23-32), 10 older children (ages 15-17), and 13 younger children (ages 11-13) participated in this study; all were female to avoid potential gender differences (Vollmer et al., 2010). The protocol was approved by the University of Southern California institutional review board, and informed assents and consents, as appropriate, were obtained from all the subjects.

\section{Experimental setup and protocol}

Contraction time. An experimental setup described previously (Kutch et al., 2010) measured high-precision index finger forces and electromyographic (EMG) signals from the first dorsal interosseous to estimate muscle contraction time using the electromyographicweighted averaging approach (EWA). Subjects were seated with their dominant arm supported by a platform, the forearm in mid prone, index finger in a few degrees of flexion and $0^{\circ}$ of metacarpophalangeal abduction, the remaining fingers wrapped around a dowel. A single differential EMG electrode (Delsys) was placed on the first dorsal interosseous (FDI). Subjects were given visual feedback while applying isometric abduction force with their index finger against a 6 degrees-of-freedom load cell (JR3). Their target force was $2 \mathrm{~N} \pm 0.5$, to be maintained as steady as possible for $20-25 \mathrm{~s}$, which was $<5 \%$ of the maximum isometric voluntary contraction for all subjects. Adult subjects also abducted with a target force of $4 \mathrm{~N} \pm 0.5$.

Dynamic manipulation ability and maximal pinch strength. To test dynamic manipulation abilities, subjects were asked to use their dominant index and thumb to compress an instrumented slender spring prone to buckling as far as possible, and hold for at least $3 \mathrm{~s}$. This spring, based on the validated Strength-Dexterity Test (Valero-Cuevas et al., 2003), becomes more unstable as it is compressed, requiring faster control of fingertip force direction with increasing compression making it impossible to compress fully (Venkadesan et al., 2007; Dayanidhi et al., 2013). The maximal level of compression $(\sim 2-3 \mathrm{~N})$ is therefore a surrogate for the maximal level of instability that can be controlled and therefore a measure of dynamic manipulation ability. Two single-axis miniature load cells (ELB4-10, Measurement Specialties) mounted at the ends of the spring measured the compression force. Maximal tipto-tip pinch forces were measured on the selfreported dominant hand using a pinch meter (B\&L Engineering). One child only completed the contraction time component of the experiment.

\section{Data reduction and analysis}

EWA is a noninvasive technique to extract the average contractile properties of an ensemble of motor units (Kutch et al., 2010). After visually selecting $20-25$ s of steady-state periods of index finger abduction force, we cross-correlate it with the accompanying full-wave rectified FDI EMG data to produce the EWA waveform. The EWA waveform is strikingly similar to the waveform produced by spike-triggered averaging (STA), a standard technique to estimate motor unit contractile properties based on intramuscular recordings (Milner-Brown et al., 1973a; Thomas et al., 1986; Thomas, 1997). To quantify muscle contraction time (CT), therefore, we used the time taken to reach the peak of this waveform, as is done in STA.

For the dynamic manipulation task, we identified the average maximal level of compression as described previously (Venkadesan et al., 2007; Dayanidhi et al., 2013). For each subject, the mean of the force during the sustained compression phases was calculated for all their attempts, and three maximum values were averaged to create a metric for dynamic manipulation ability, which we call the dynamic manipulation score (DMS), in units of grams of force (gmf).

Age was the independent variable for our statistical analysis, either divided into bins $(11-13,15-17,23-32$ years) for ANOVAs or as a con- 
tinuous variable for regression analyses. Oneway ANOVAs tested for differences among age groups in mean contraction time, DMS, pinch strength, and firing rates of the raw EMG (median frequency and lowest nonzero autocorrelation time lag). A pairwise $t$ test tested for differences in firing rates between the $2 \mathrm{~N}$ and $4 \mathrm{~N}$ contraction times task in adults. Last, we performed linear regressions between contraction time and dynamic manipulation score, and among contraction time and pinch strength.

\section{Results}

Regarding manipulation ability (Fig. 1A), adults achieved higher DMS than children (Fig. $1 B$; representative data from two subjects), and DMS increased as a function of age (Fig. 1C; raw histograms, see statistical analysis below): group median DMS values of 122, 176, and $200 \mathrm{gmf}$ for young children, older children, and adults, respectively.

Muscle contraction time (Fig. 1C) showed a complementary trend with age. Young children had longer CT values than adults (Fig. 1E; data from the same two representative subjects), and CT decreased as a function of age (Fig. $1 F$; raw histograms, see statistical analysis below): the group median CT values were 74, 54, and 49 ms for young children, older children, and adults, respectively.

When treating age as a continuous independent variable, a significant association was seen with DMS (Fig. 2, green squares; $p<0.0001$ ) and CT (Fig. 2, blue circles; $p<0.005$ ). A one-way ANOVA for DMS showed similar trends among age bins: highly significant differences $(p<0.00001)$ among adults and children (Fig. 3, right), with younger children having a mean value of $125.05 \pm 19.25 \mathrm{gmf}$, older children $166.81 \pm 20.19 \mathrm{gmf}$, and adults having a mean value of $199.06 \pm 19.84 \mathrm{gmf}$, ignoring the one outlier. Post hoc Bonferroni tests revealed significant differences among all three age groups. A one-way ANOVA for CT showed significant differences $(p<0.01)$ between adults and children (Fig. 3, right), with young children having a mean time of $66.54 \pm 12.4 \mathrm{~ms}$, older children of $56.25 \pm 8.7 \mathrm{~ms}$, and adults of $52.12 \pm 9.6 \mathrm{~ms}$. Post hoc Bonferroni tests revealed differences between the young children and adults, but not between older children and adults. Two children had values that were detected to be outliers on a boxplot (i.e., $>1.5$ times the interquartile range) and not included in the analysis (Fig. 3). Including the outliers made the results of the ANOVA more significant $(p<$ 0.005), and the post hoc tests showed differences between the young and older children as well.

A significant negative association was observed between CT and DMS using linear regression (Fig. 4; $p<0.005, r^{2}=0.28, r=$ $0.53)$. Every $10 \mathrm{~ms}$ decrease in CT was associated with an increase in DMS of $\sim 20$ gmf.

As expected, strength increased with age. Significant differences were seen for the tip-to-tip pinch $(p<0.00005)$, with young children having a mean of $3542 \pm 629$ gmf, older children $4150 \pm 784 \mathrm{gmf}$, and adults $5308 \pm 879 \mathrm{gmf}$. Regression analysis showed that CT had a significant negative association with tipto-tip pinch $\left(p<0.05, r^{2}=0.16\right)$. The fingers used, and their configuration, is the same for the dynamic manipulation and the tip-to-tip pinch tasks.
We also investigated the potential confound of different firing rates across age groups. Given their lower strength, we would expect children to have their muscles operating at a higher firing rate, for a given force, compared with the adults. The median frequency of firing of the FDI EMG was higher in younger children $(97.04 \pm 12.16 \mathrm{~Hz})$ compared with adults $(90 \pm 6.88 \mathrm{~Hz})$, which tended to significance $(p=0.07)$. Exclusion of one adult outlier, detected using a boxplot, revealed significant differences $(p<0.05)$. As a complementary analysis of median frequency, the time of the lowest nonzero autocorrelation peak was significantly $(p<0.0005)$ different, with younger children showing a shorter lag of $64.29 \pm 6.96$ $\mathrm{ms}$ and adults a longer one of $84.6 \pm 10.6 \mathrm{~ms}$. Consequently, these analyses reveal that children were operating at a higher firing rate compared with adults.

To evaluate the effect of higher firing rates on $\mathrm{CT}$, we performed the task in adults at $2 \mathrm{~N}$ and $4 \mathrm{~N}$ target force levels. A pairwise $t$ test revealed a significant increase in median frequency in adults between $2 \mathrm{~N}$ and $4 \mathrm{~N}(90 \pm 6.88 \mathrm{vs} 98.53 \pm$ $14.6 \mathrm{~Hz}, p<0.05$ ) with the adults at $4 \mathrm{~N}$ target force firing at a similar higher rate as the children at $2 \mathrm{~N}$. A pairwise $t$ test also revealed significant differences in autocorrelation time lag in adults when comparing the $2 \mathrm{~N}$ and $4 \mathrm{~N}$ target force $(84.6 \pm$ 10.6 vs $63.79 \pm 15.71 \mathrm{~ms}, p<0.001)$, again the $4 \mathrm{~N}$ was similar to the time lag in children at $2 \mathrm{~N}$. However, no significant differences were seen in the CT for the adults when comparing the $2 \mathrm{~N}$ and $4 \mathrm{~N}$ target force $(52.12 \pm 9.6$ vs $54.97 \pm 12.6, p=$ $0.26)$. This supports the assertion that CT as estimated by EWA at these low force levels is not sensitive to motor unit firing rate. These analyses therefore confirm that younger children had a higher average firing rates in the motor units compared with adults in a muscle generating approximately the same force, but that increasing the motor unit firing rates does not increase the contraction time. Therefore, the results in our comparison of contraction times between children and adults cannot be attributed to differences in firing rates. 


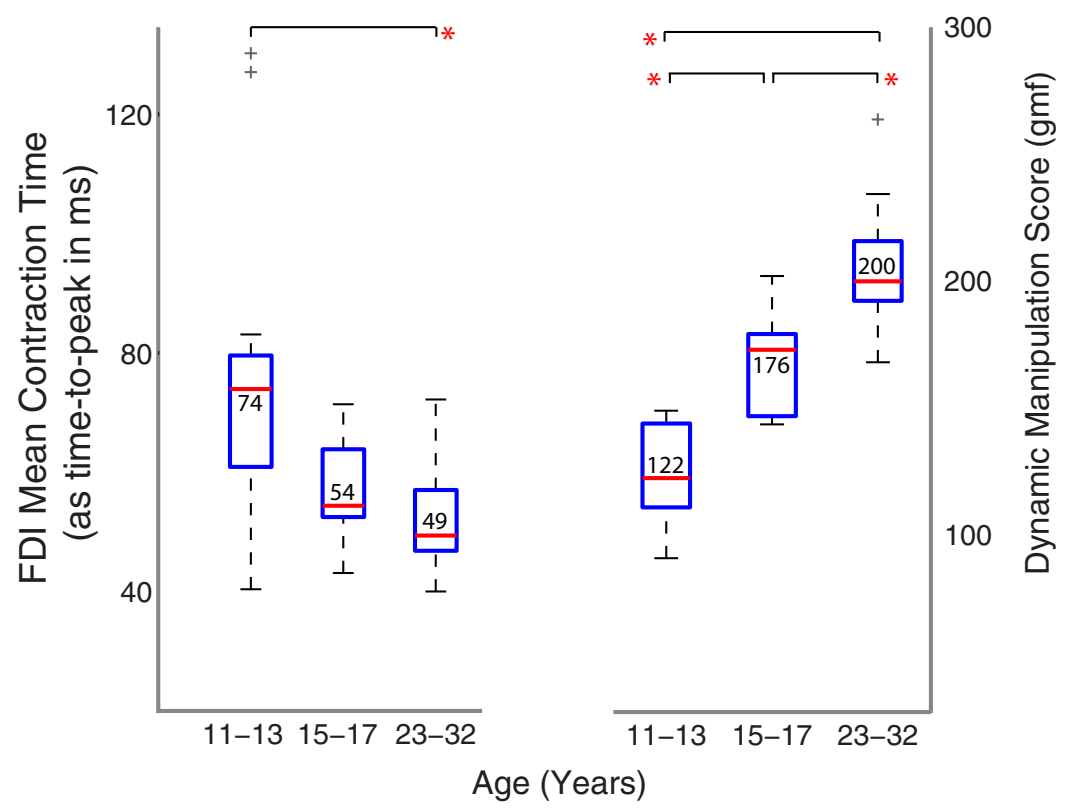

Figure 3. Change in dynamic manipulation (right) and contraction time (left) across the age groups. *Significant differences $(p<0.01)$ are based on one-way ANOVAs across the age groups and show significant changes for both contraction time as well as dynamic manipulation abilities. The contraction time was measured in terms of the average time to peak (i.e., longer time translates to slower contraction time and vice versa). The outliers are indicated by gray crosshairs.

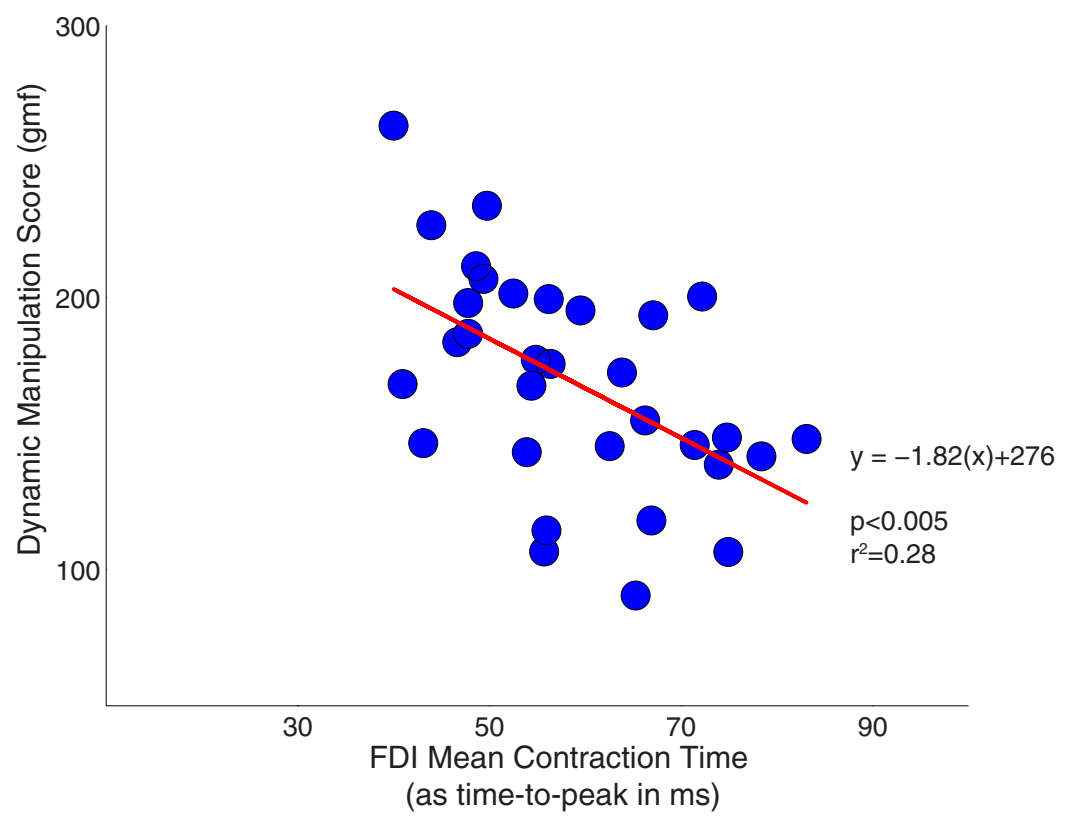

Figure 4. Relationship between contraction time of the FDI and dynamic manipulation abilities. The regression line was significantly different from zero $(p<0.005)$, indicating an association between these two variables with $28 \%$ shared variance. The contraction time was measured in terms of the average time to peak (i.e., longer time translates to slower contraction time and vice versa).

\section{Discussion}

We found evidence that contraction time of intrinsic hand muscles decreases as an individual transitions from childhood to adulthood, which for the first time demonstrates developmental improvements in the dynamics of the human peripheral motor system that could facilitate the fine control required for dynamic manipulation. Correspondingly, a significant association was observed between muscle contraction time and dynamic manipulation score. Although we naturally saw the expected gains in pinch strength, it only shows half the association with contraction time as the dynamic manipulation score; and surprisingly, the changes in dynamic manipulation scores and muscle contraction time were measured at very low force levels. With certain limitations discussed below, these findings critically extend our understanding of central and peripheral contributors to the development of dynamic manipulation.

Although development of noninvasive techniques is required to disambiguate and clarify the role of the central and peripheral factors to the development of manipulation tasks, it is important here to discuss some of the assumptions and limitations. EWA and the related widely used spike-triggered average (STA) technique share the limitation that their estimate for the contraction time of a motor unit twitch is firing-rate dependent. In general, the STA/EWA estimate for contraction time will decrease with increased firing rate (Calancie and Bawa, 1986; Lim et al., 1995). However, this limitation serves to strengthen our interpretation. It could be argued that the young children had an EWA-estimated CT that was trivially different from adults simply because they were operating at a different percentage of their maximum force, and hence a different average motor unit firing rate. Because the children were demonstrably weaker, we can assume that children must be operating at a higher firing rate to achieve the same force output, an assumption supported by analyses of the EMG power spectrum and autocorrelation. Therefore, this higher firing rate could only serve to make the estimate of CT lower in children compared with adults, which is the opposite of our data. Furthermore, we tested this interpretation by having adults increase their force output from $2 \mathrm{~N}$ to $4 \mathrm{~N}$, forcing the adults to operate at a higher percentage of their maximum and simulating the weaker muscle of the children. We did not observe increases in EWAestimated CT, arguing that the longer CT observed in children is not a trivial consequence of firing rate.

Successful dynamic manipulation depends on the ability to quickly control the fingertip force magnitude and direction (Murray et al., 1994; Valero-Cuevas et al., 2003), which in turn requires quickness of response in both the controller (i.e., the neural pathways) and the actuators (i.e., the muscles). Even at low force magnitudes, such as in this study, controlling a dynamically unstable object requires the ability to direct fingertip force vectors appropriately (Venkadesan et al., 2007). Consequently, success at this task requires sufficient controller and actuator response time to respond quickly, more so in the presence of noise (Faisal et al., 
2008). Although the central conduction time of the corticospinal tract has been shown by TMS to reach maturity by the age of 13 (Nezu et al., 1997), this effectively represents the speed in a minimally mature corticospinal tract. During the adolescent years, there is still ongoing development of the corticospinal, frontoparietal, and cerebellar tracts associated with dynamic manipulation skills (Paus et al., 1999; Lebel et al., 2008), which can allow for better parallel processing. There are microstructural changes in the corticospinal tract (Lebel et al., 2008), with increased organization and myelination seen as higher fractional anisotropy leading to an increase in size of the pathway. This is further supported by evidence that the fractional anisotropy in adult piano players is significantly greater, related to the amount of practice (Bengtsson et al., 2005) and thus shows an influence of activitydependent processes on structural maturation. Here we show, for the first time, that there are also changes in the response time of the muscle actuator, evidenced by decrease in the contraction time of the FDI that has a significant association with improvements in dynamic manipulation score.

In our study, the contraction time computed using the EWA is based on a cross-correlation between force and EMG waveforms (Kutch et al., 2010) and is consequently related to some change in the peripheral muscular apparatus (i.e., the actuator response time). This leads us to propose that perhaps the observed differences in twitch properties are related to improved $\mathrm{Ca}^{2+}$ uptake by the sarcotubules (Brody, 1976) in adulthood. Moreover, in other animals, shorter muscle contraction times in superfast muscles, such as a toadfish bladder, are related to this $\mathrm{Ca}^{2+}$ pump and parvalbumin concentration, along with unbinding of the $\mathrm{Ca}^{2+}$ from troponin $\mathrm{C}$ and faster cross-bridge detachment (Rome et al., 1996). Our results are similar, in part, to the results from postnatal development in mammals (Close, 1964), where there is a $50-80 \%$ improvement in contraction time, possibly related to the significant increase in parvalbumin concentration $(\sim 500 \%)$, secondary to neural activation of the muscles (Leberer and Pette, 1986).

Here we show that, although finger configuration in tip-to-tip pinch is similar to that during the measurement of dynamic manipulation, it only shows half the association with contraction time as the dynamic manipulation score (16\% vs $28 \%$ ). Consequently, it does not appear that it benefits maximal voluntary force capabilities in general but could potentially decrease the time in which it is reached. A prior version of this test has been shown to capture a unique trait of dynamic fingertip force coordination, which is different from pinch strength in typically developing children through adolescence (Vollmer et al., 2010). Moreover, pinch strength is a poor predictor of dynamic manipulation abilities per se given that both experimental and analytical results show that muscle strength (which can be used to stiffen the fingers) does not help achieve higher levels of performance during these unstable dynamical tasks (Venkadesan et al., 2007; Dayanidhi et al., 2013).

Our results have important implications for uncovering the mechanisms of deficits in, and therapeutic options for, dynamic manipulation in atypical development, such as in children with cerebral palsy. Although muscular atrophy and consequently weakness are observed, there are no differences in motor unit recruitment characteristics at low force levels (Rose and McGill, 2005). Manipulation tasks do not require high amounts of force (Smaby et al., 2004), and deficits in hand function cannot be explained purely by central contributors. Recent evidence suggests that extended practice during developmentally critical periods, such as in the adolescent years, is effective for improving hand function (Gordon et al., 2006), secondary to neural plasticity (Armand et al., 1997). Here we propose that improvements in peripheral factors of contraction time might complement these findings.

\section{References}

Armand J, Olivier E, Edgley SA, Lemon RN (1997) Postnatal development of corticospinal projections from motor cortex to the cervical enlargement in the macaque monkey. J Neurosci 17:251-266. Medline

Bengtsson SL, Nagy Z, Skare S, Forsman L, Forssberg H, Ullén F (2005) Extensive piano practicing has regionally specific effects on white matter development. Nat Neurosci 8:1148-1150. CrossRef Medline

Brody IA (1976) Regulation of isometric contraction in skeletal muscle. Exp Neurol 50:673-683. CrossRef Medline

Calancie B, Bawa P (1986) Limitations of the spike-triggered averaging technique. Muscle Nerve 9:78-83. CrossRef Medline

Chan KM, Doherty TJ, Brown WF (2001) Contractile properties of human motor units in health, aging, and disease. Muscle Nerve 24:1113-1133. CrossRef Medline

Close R (1964) Dynamic properties of fast and slow skeletal muscles of the rat during development. J Physiol 173:74-95. Medline

Dayanidhi S, Hedberg $\AA$, Valero-Cuevas FJ, Forssberg H (2013) The developmental improvements in dynamic control of fingertip forces last throughout childhood and into adolescence. J Neurophysiol. Advance online publication. Retrieved July 17, 2013. doi: 10.1152/jn.00320.2013. CrossRef Medline

Faisal AA, Selen LP, Wolpert DM (2008) Noise in the nervous system. Nat Rev Neurosci 9:292-303. CrossRef Medline

Forssberg H, Eliasson AC, Kinoshita H, Johansson RS, Westling G (1991) Development of human precision grip: I. Basic coordination of force. Exp Brain Res 85:451-457. Medline

Giedd JN, Blumenthal J, Jeffries NO, Castellanos FX, Liu H, Zijdenbos A, Paus T, Evans AC, Rapoport JL (1999) Brain development during childhood and adolescence: a longitudinal MRI study. Nat Neurosci 2:861-863. CrossRef Medline

Gordon AM, Charles J, Wolf SL (2006) Efficacy of constraint-induced movement therapy on involved upper-extremity use in children with hemiplegic cerebral palsy is not age-dependent. Pediatrics 117:e363e373. CrossRef Medline

Häger-Ross C, Rösblad B (2002) Norms for grip strength in children aged 4-16 years. Acta Paediatr 91:617-625. CrossRef Medline

Heizmann CW, Berchtold MW, Rowlerson AM (1982) Correlation of parvalbumin concentration with relaxation speed in mammalian muscles. Proc Natl Acad Sci U S A 79:7243-7247. CrossRef Medline

Kutch JJ, Kuo AD, Rymer WZ (2010) Extraction of individual muscle mechanical action from endpoint force. J Neurophysiol 103:3535-3546. CrossRef Medline

Lebel C, Walker L, Leemans A, Phillips L, Beaulieu C (2008) Microstructural maturation of the human brain from childhood to adulthood. Neuroimage 40:1044-1055. CrossRef Medline

Leberer E, Pette D (1986) Neural regulation of parvalbumin expression in mammalian skeletal muscle. Biochem J 235:67-73. Medline

Lim KY, Thomas CK, Rymer WZ (1995) Computational methods for improving estimates of motor unit twitch contraction properties. Muscle Nerve 18:165-174. CrossRef Medline

Martin JH (2005) The corticospinal system: from development to motor control. Neuroscientist 11:161-173. CrossRef Medline

Milner-Brown HS, Stein RB, Yemm R (1973a) The contractile properties of human motor units during voluntary isometric contractions. J Physiol 228:285-306. Medline

Milner-Brown HS, Stein RB, Yemm R (1973b) The orderly recruitment of human motor units during voluntary isometric contractions. J Physiol 230:359-370. Medline

Murray RM, Sastry SS, Zexiang L (1994) A mathematical introduction to robotic manipulation. Boca Raton, FL: CRC.

Nezu A, Kimura S, Uehara S, Kobayashi T, Tanaka M, Saito K (1997) Magnetic stimulation of motor cortex in children: maturity of corticospinal pathway and problem of clinical application. Brain Dev 19:176-180. CrossRef Medline

O'Brien TD, Reeves ND, Baltzopoulos V, Jones DA, Maganaris CN (2010) In vivo measurements of muscle specific tension in adults and children. Exp Physiol 95:202-210. CrossRef Medline

Olivier E, Edgley SA, Armand J, Lemon RN (1997) An electrophysiological 
study of the postnatal development of the corticospinal system in the macaque monkey. J Neurosci 17:267-276. Medline

Paus T, Zijdenbos A, Worsley K, Collins DL, Blumenthal J, Giedd JN, Rapoport JL, Evans AC (1999) Structural maturation of neural pathways in children and adolescents: in vivo study. Science 283:1908-1911. CrossRef Medline

Rome LC, Syme DA, Hollingworth S, Lindstedt SL, Baylor SM (1996) The whistle and the rattle: the design of sound producing muscles. Proc Natl Acad Sci U S A 93:8095-8100. CrossRef Medline

Rose J, McGill KC (2005) Neuromuscular activation and motor-unit firing characteristics in cerebral palsy. Dev Med Child Neurol 47:329-336. CrossRef Medline

Smaby N, Johanson ME, Baker B, Kenney DE, Murray WM, Hentz VR (2004) Identification of key pinch forces required to complete functional tasks. J Rehabil Res Dev 41:215-224. CrossRef Medline

Thomas CK (1997) Contractile properties of human thenar muscles paralyzed by spinal cord injury. Muscle Nerve 20:788-799. CrossRef Medline

Thomas CK, Ross BH, Stein RB (1986) Motor-unit recruitment in human first dorsal interosseous muscle for static contractions in three different directions. J Neurophysiol 55:1017-1029. Medline

Thomas CK, Johansson RS, Westling G, Bigland-Ritchie B (1990) Twitch properties of human thenar motor units measured in response to intraneural motor-axon stimulation. J Neurophysiol 64:1339-1346. Medline

Valero-Cuevas FJ, Smaby N, Venkadesan M, Peterson M, Wright T (2003) The strength-dexterity test as a measure of dynamic pinch performance. J Biomech 36:265-270. CrossRef Medline

Venkadesan M, Valero-Cuevas FJ (2008) Neural control of motion-to-force transitions with the fingertip. J Neurosci 28:1366-1373. CrossRef Medline

Venkadesan M, Guckenheimer J, Valero-Cuevas FJ (2007) Manipulating the edge of instability. J Biomech 40:1653-1661. CrossRef Medline

Vollmer B, Holmström L, Forsman L, Krumlinde-Sundholm L, ValeroCuevas FJ, Forssberg H, Ullén F (2010) Evidence of validity in a new method for measurement of dexterity in children and adolescents. Dev Med Child Neurol 52:948-954. CrossRef Medline 